\title{
Stroke Risk Among Non-Elderly Users of Haloperidol or First-Generation Antipsychotics vs Second-Generation Antipsychotics: A Cohort Study from a US Health Insurance Claims Database
}

\author{
Daniel Fife ${ }^{1}\left[\right.$ Clair Blacketer $^{1} \cdot$ Karl Knight $^{2} \cdot$ James Weaver $^{1}$
}

Accepted: 27 May 2021 / Published online: 9 June 2021

(c) The Author(s) 2021

\begin{abstract}
Background Previous studies have reported an increased risk of stroke in patients taking antipsychotics. However, most of these studies have been conducted in the elderly population.

Objective We estimated stroke risk in new users of any first-generation antipsychotic or haloperidol, vs second-generation antipsychotics among patients aged 18-64 years without a recent dementia diagnosis and, separately, regardless of a recent dementia diagnosis.

Methods Data were obtained from IBM MarketScan ${ }^{\circledR}$ Commercial Database (1 January, 2001-31 December, 2017). Among new users without a recent dementia diagnosis, stroke risk for first-generation antipsychotics (FGAw/oD cohort) or haloperidol (HALw/oD cohort) was compared with second-generation antipsychotics (SGAw/oD cohort). A similar comparison was conducted among new users regardless of dementia diagnosis: first-generation antipsychotics (FGA cohort) or haloperidol (HAL cohort) vs second-generation antipsychotics (SGA cohort). Crude incident stroke rates within each cohort were determined. For hazard ratios, three propensity score matching strategies were used: unadjusted (crude), Sentinel propensity score strategy, and large-scale regularized regression model (adapted propensity score strategy).

Results Each cohort included $\geq 12,000$ patients. The incident rates for stroke per 1000 person-years were 3.10 (FGAw/oD), 5.99 (HALw/oD), 0.85 (SGAw/oD), 3.14 (FGA), 6.12 (HAL), and 0.90 (SGA). Pre-planned analysis with adapted propensity score strategy matching yielded calibrated hazard ratios for stroke: FGAw/oD vs SGAw/oD: 2.05 (calibrated confidence interval 1.13-3.89); HALw/oD vs SGAw/oD: 2.47 (1.14-5.48), FGA vs SGA: 1.64 (0.94-2.97), and HAL vs SGA: 1.98 (0.99-4.00). A post-hoc sensitivity analysis to address potential bias introduced by the 2015 change from the International Classification of Diseases, Ninth Revision to the International Classification of Diseases, Tenth Revision yielded calibrated hazard ratios for FGAw/oD vs SGAw/oD: 1.59 (0.87-3.01), HALw/oD vs SGAw/oD: 2.79 (1.24-6.42), FGA vs SGA: 1.41 (0.79-2.62), and HAL vs SGA: 3.47 (1.63-7.92).
\end{abstract}

Conclusions Among adults aged $\leq 64$ years, without a recent dementia diagnosis, stroke risk is higher among those exposed to haloperidol compared with those exposed to second-generation antipsychotics.

Daniel Fife

DFife@its.jnj.com

1 Department of Epidemiology, Janssen Research \&

Development, LLC, 1125 Trenton-Harbourton Road,

Titusville, NJ 08560, USA

2 Established Products, Janssen Research \& Development,

LLC, Titusville, NJ, USA 


\section{Key Points}

Previous studies have reported an increased risk of stroke in patients taking antipsychotics. However, most of these studies have been conducted in the elderly population.

We estimated and compared the risk of stroke among new users of first-generation antipsychotics and haloperidol with new users of second-generation antipsychotics in non-elderly patients (aged $\leq 64$ years) without a recent dementia diagnosis and, separately, regardless of a recent dementia diagnosis. We observed that stroke risk was elevated among non-elderly patients without a recent dementia diagnosis taking haloperidol compared with patients using second-generation antipsychotics.

\section{Introduction}

Antipsychotics, first generation and second generation, are approved for the treatment of schizophrenia, schizoaffective disorder, bipolar disorder, hyperactivity, severe behavioral problems, agitation, psychotic disorders, Tourette syndrome, generalized nonpsychotic anxiety, major depressive disorder (adjunctive treatment), and autism-associated irritability [1]. In the USA, the number of prescriptions for psychiatric conditions has increased substantially among adults [3-5], with a considerable proportion of these prescriptions being for off-label indications [6]. There is substantial evidence that patients who use antipsychotics are at increased risk of stroke [7-10], as well as other life-threatening or fatal medical events such as pneumonia, hip fracture, thromboembolism, sudden cardiac death, and myocardial infarction [11]. However, it is difficult to adequately address the potentially relevant confounders and compare the stroke risks associated with different antipsychotics or classes of antipsychotics because users of various antipsychotics may differ in health habits, economic status, comorbid conditions, and concurrent medications. Indeed, some studies suggest a greater risk of stroke among users of first-generation antipsychotics as compared with second-generation antipsychotics [10, 12]. Shin et al. reported that among elderly patients, haloperidol, a first-generation antipsychotic, was associated with a greater risk of stroke as compared with risperidone, a second-generation antipsychotic [13]. However, other studies have suggested a similar risk between first-generation and second-generation antipsychotics [7, 12, 14] or a greater risk with second-generation antipsychotics $[15,16]$.
Though strokes are more common among elderly individuals than among the young individuals [17], more than half of antipsychotic users are $<65$ years old [18, 19] and though many studies evaluating antipsychotics and the risk of stroke have been conducted in the elderly population [13, 20-22], relatively few have been conducted in younger populations. Mundet-Tuduri et al. [8] reported a higher prevalence of stroke in adult patients using antipsychotics compared with non-users (odds ratio 2.33). Another recent retrospective study conducted using the Sentinel database in the non-elderly population without dementia showed a hazard ratio (HR) for stroke of $1.75,95 \%$ confidence interval (CI 1.17-2.63) among users of first-generation antipsychotics relative to second-generation antipsychotics and 1.80 (0.93-3.48) among users of haloperidol relative to second-generation antipsychotics, before propensity score (PS) matching. After PS matching, the HRs were 0.87 (0.54-1.41) and $1.31(0.53-3.21)$, respectively [12]. We have described the risk of stroke among new users of firstgeneration antipsychotics and haloperidol vs new users of second-generation antipsychotics among elderly patients, regardless of dementia diagnosis, in a separate report [23].

The present study was conducted to: (1) replicate the findings of the Sentinel study [12] using a different database and PS matching mimicking that of the Sentinel study and (2) to extend those findings by matching on a more comprehensive adapted (large-scale) PS. We estimated and compared the risk of stroke among new users of first-generation antipsychotics and haloperidol vs new users of second-generation antipsychotics in non-elderly patients without a recent dementia diagnosis and, separately, regardless of a recent dementia diagnosis. We stratified on dementia to mimic the approach adopted by the Sentinel study, and because the label of haloperidol, which differentiates between elderly and non-elderly populations, has a black-box warning for dementia [24].

\section{Methods}

\subsection{Data Sources}

Data for this study were obtained from the IBM MarketScan ${ }^{\circledR}$ Commercial Database (CCAE), a large, nationally representative, US health claims database of patients $<65$ years of age that includes data for active employees, early retirees, Consolidated Omnibus Budget Reconciliation Act continuers, and their dependents who are insured by employersponsored plans. The database includes information on demographic characteristics; outpatients' prescription drugs, diagnoses, and procedures; and hospitalizations including discharge diagnoses for 142 million beneficiaries between January 2000 and August 2018. The use of CCAE was 
reviewed by the New England Institutional Review Board and determined to be exempt from broad institutional review board approval, as this research project did not involve human patient research.

\subsection{Study Design and Study Population}

This retrospective comparative cohort study included data available from 1 January, 2001 through 31 December, 2017 with the earliest cohort entry starting 1 July, 2001. Prior data from 2001 contributed information on the conditions required for cohort inclusion. Thus, the start and end dates were similar to those of the Sentinel study (1 January, 2001-30 September, 2015).

The original CCAE database has been converted to the observational medical outcomes partnership Common Data Model [25, 26] to allow for consistent analyses using standardized analytic and statistical software [27-29]. This model standardizes the structure and content of data by mapping coding systems such as the International Classification of Diseases, Ninth Revision, Clinical Modification (ICD9-CM) and the International Classification of Diseases, Tenth Revision, Clinical Modification (ICD-10-CM) to Systematized Nomenclature of Medicine - Clinical Terms (SNOMED-CT) concepts by leveraging relationships that have been curated by the Unified Medical Language System [30]. The SNOMED-CT defines cohorts by walking up and down the comprehensive hierarchy. The relationship between SNOMED-CT and ICD-9-CM (and ICD-10-CM) is used to create the definition included in each code. In the present study, we were limited to the ICD-9-CM codes used in the Sentinel study by Taylor et al. [12] to define stroke. Furthermore, we used a similar approach and crosswalked from ICD-9-CM to SNOMED-CT. The relationships between SNOMED-CT and ICD-10-CM were examined. As we did not have a reference from Taylor et al. [12] for the ICD-10-CM codes to be used, we relied on the mappings provided by the Unified Medical Language System. These ICD-10-CM codes were used for the incidence comparison (Electronic Supplementary Material [ESM]). As these figures indicated a change in incidence estimates after $30 \mathrm{Sep}-$ tember, 2015, we conducted a post-hoc analysis that was limited to the data through 30 September, 2015.

New users of first-generation antipsychotics, haloperidol, or second-generation antipsychotics aged 18-64 years were included in the study cohort when they first met all the following conditions: received a dispensing of a first-generation or second-generation antipsychotic, had at least 183 days of continuous enrollment before that dispensing, had no diagnosis of stroke or cancer during the 183 days up to and including the dispensing date, and during the 183 days before that dispensing had received no dispensing of a first-generation or second-generation antipsychotic. The dispensing date was the patient's index date. Patients who were exposed to both first-generation and second-generation antipsychotics on their index date were excluded.

We focused on four new user target cohorts (FGAw/oD: first-generation antipsychotics without a recent dementia diagnosis, i.e., without a dementia diagnosis in the 183 days prior to entering the cohort, HALw/oD: haloperidol without a recent dementia diagnosis, FGA: first-generation antipsychotics with or without a recent dementia diagnosis, and HAL: haloperidol with or without a recent dementia diagnosis) and two new user comparator cohorts (SGAw/oD: second-generation antipsychotics without a recent dementia diagnosis and SGA: second-generation antipsychotics with or without a recent dementia diagnosis). We compared the stroke risk in FGAw/oD and HALw/oD cohorts to the stroke risk in the SGAw/oD cohort and the stroke risk in FGA and HAL cohorts to the stroke risk in the SGA cohort. For each pairwise comparison, patients contributed "as-treated" time at risk in the cohort for which they first qualified from the day after they first entered that cohort until the first occurrence among: receiving a medication associated with the other cohort, having the study outcome (see below), having a gap of $>30$ days in the supply of the cohort-defining drug (> 30 days from the end of the days' supply to the next dispensing), reaching the end of insurance enrollment, or reaching the end of the study period.

As in the Sentinel study [12], and using the same code list for stroke as did that study, stroke as the study outcome was defined by a diagnosis code for stroke as the primary condition in the insurance claim associated with an inpatient stay, and stroke as a covariate was defined as an inpatient or outpatient diagnosis of stroke using the same code list as above. The code lists used for identifying first-generation antipsychotics, haloperidol, second-generation antipsychotics, and stroke are available in the protocol, which is pre-registered at https://clinicaltrials.gov/ct2/show/NCT04002700 and is publicly available in greater detail at https://github.com/ ohdsi-studies/StrokeRiskInApUsers/tree/master/documents.

Reimbursement coding requirements in the USA changed from ICD-9-CM to ICD-10-CM on 1 October, 2015 (ESM). We observed a decrease in the yearly incidence of stroke after 2015 associated with the change in coding from ICD9-CM to ICD-10-CM and indicating that the ICD-9-CM codes used for stroke correspond to ICD-10-CM codes that identify a clinically different patient population. Because this change appeared likely to affect the incidence of stroke in the study population, we conducted a post-hoc analysis where the study end date was redefined as 30 September, 2015. That end date for the post-hoc analysis was selected to reflect the end date of the Sentinel study [12], whose end date coincided with the switch of many US healthcare databases from ICD-9-CM to ICD-10-CM. 


\subsection{Statistical Analysis}

The crude incidence rate (IR) and incidence proportion (IP) of stroke were estimated within each exposure cohort and pre-defined sex subgroup. Incidence rate was calculated as the number of patients with the outcome during the time-atrisk window divided by the total time-at-risk in years and was reported as IR/1000 person-years. Incidence proportion was calculated as the number of patients with the outcome during the time-at-risk window divided by the total number of patients with time-at-risk and was reported as IP/1000 patients. Population-level effect estimation analyses of new users were performed using a comparative cohort design including four pairwise comparisons: FGAw/oD vs SGAw/ oD, HALw/oD vs SGAw/oD, FGA vs SGA, and HAL vs SGA.

Propensity score matching was used to reduce potential confounding because of an imbalance in baseline covariates between the target and comparator cohorts [31]. The PS adjustments are explained in detail in the ESM. A covariate balance was summarized by computing before and after PS matching, the mean value, and the associated standardized mean difference for each baseline covariate.

The HR for each outcome during the time at risk was estimated by applying a Cox proportional hazards model conditioned on the PS-matched sets. For each outcome model, the uncalibrated and empirically calibrated $\mathrm{HR}, 95 \% \mathrm{CI}$, and $p$-value [34] were reported. The method used for empirical calibration is reported in the ESM. For both cohorts being compared, patients were required to have at least 1 day of continuous observation after the time-at-risk start. The timeto-event of the outcome was determined by calculating the number of days from start of the time-at-risk window to the date of the outcome event. Although many hypotheses were tested, no correction for multiple testing was made because doing so may reduce the sensitivity for detecting potential adverse events [35].

\section{Results}

Results of the present study are publicly available through the interactive online tool at https://data.ohdsi.org/Strok eRiskInNonElderlyApUsers/.

\subsection{Baseline Characteristics}

Patients initially identified for inclusion in each cohort were: FGAw/oD cohort: 50,864, HALw/oD cohort: 12,472, FGA cohort: 51,473; HAL cohort: 12,898, SGAw/oD cohort: 926,152, and SGA cohort: 932,845. The slightly lower counts after limiting the cohorts to patients with at least 1 day at risk after exposure are shown in Table 1. Differences between the cohorts exposed to first-generation antipsychotics and the cohorts exposed to second-generation antipsychotics before and after matching are shown in Table 1, ESM, and Figs. 1, 2, 3 and 4. These figures demonstrate the greatly improved covariate balance after matching and indicate that the adapted PS matching strategy addressed the covariate imbalance to a greater extent than the Sentinel PS matching strategy.

\subsection{Crude First Post-Index Stroke Incidence}

The crude IR for stroke per 1000 person-years was 3.10 for patients of the FGAw/oD cohort, 5.99 for the HALw/oD cohort, 3.14 for FGA cohort, 6.11 for HAL cohort, 0.85 for the SGAw/oD cohort, and 0.90 for the SGA cohort during the pre-planned analyses. These IRs were similar in the posthoc analyses (Table 2).

\subsection{PS-Adjusted HR for First Post-Index Stroke}

Table 3 reports HRs for the study comparisons and Figs. 1, 2, 3 and 4 report covariate balance diagnostics that correspond with each HR. A HR that corresponds with a covariate balance diagnostic where the after-matching standardized mean differences are low is less likely to be affected by observed confounding than where after-matching standardized mean differences are high. The HR for stroke for patients of the FGAw/oD vs SGAw/oD cohort in the pre-planned analysis was 2.75 (95\% CI 1.27-6.59) with the Sentinel PS matching strategy (Fig. 1A) and the cHR was 2.05 (95\% CI 1.13-3.89) with the adapted PS matching strategy (Fig. 1B). The HR of stroke in patients of the HALw/oD vs SGAw/oD cohort was $1.80(0.62-5.86)$ with Sentinel PS matching (Fig. 2A) and the cHR was 2.47 (1.14-5.48) with the adapted PS matching strategy (Fig. 2B). The HR for stroke in patients of the FGA vs SGA cohort was 2.18 (1.09-4.64) with the Sentinel PS matching strategy (Fig. 3A) and the cHR was 1.64 (0.94-2.97) with the adapted PS matching strategy (Fig. 3B). The HR for stroke in patients of the HAL vs SGA cohort was $2.20(0.81-6.98)$ with the Sentinel PS matching strategy (Fig. 4A) and 1.98 (0.99-4.00) with the adapted PS matching strategy (Fig. 4B). Results of the post-hoc analysis are also presented in Table 3.

\section{Discussion}

In the present study, among patients without a recent dementia diagnosis (the population analyzed in the Sentinel study), the crude (unadjusted) HR for stroke for first-generation vs second-generation antipsychotics and for haloperidol vs second-generation antipsychotics had point estimates $>1$ and CIs that excluded 1 in both the pre-planned and post-hoc 


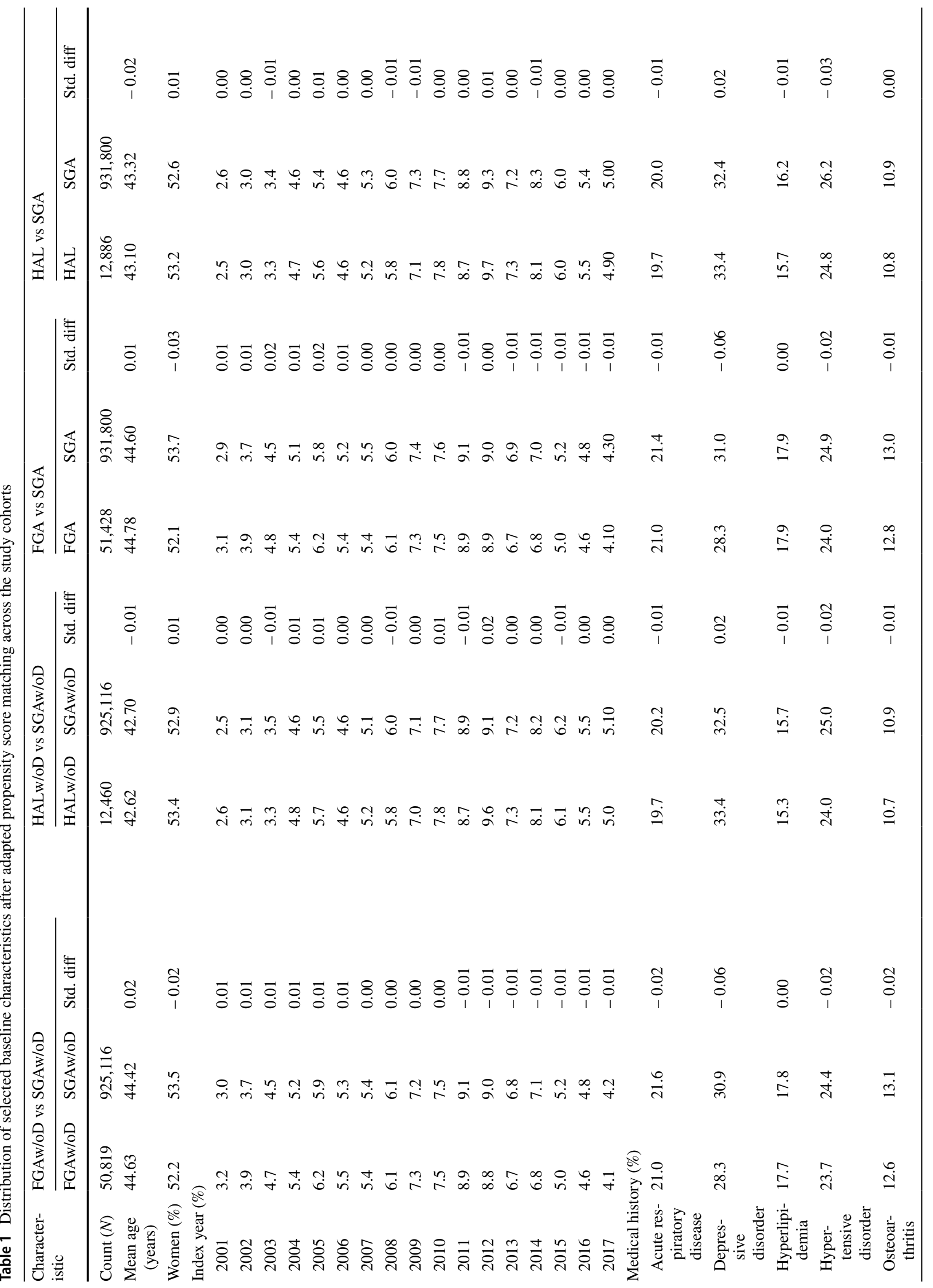




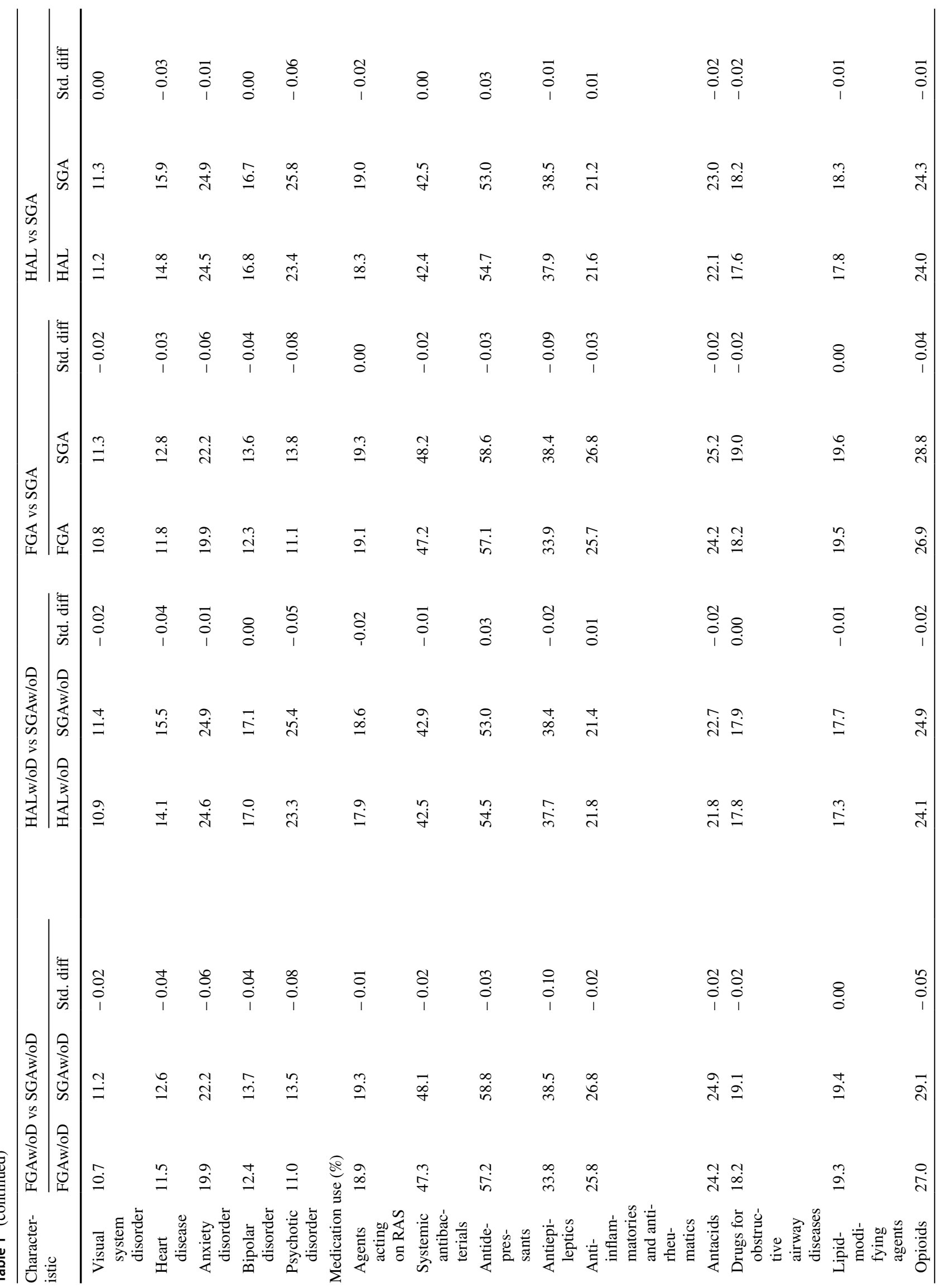




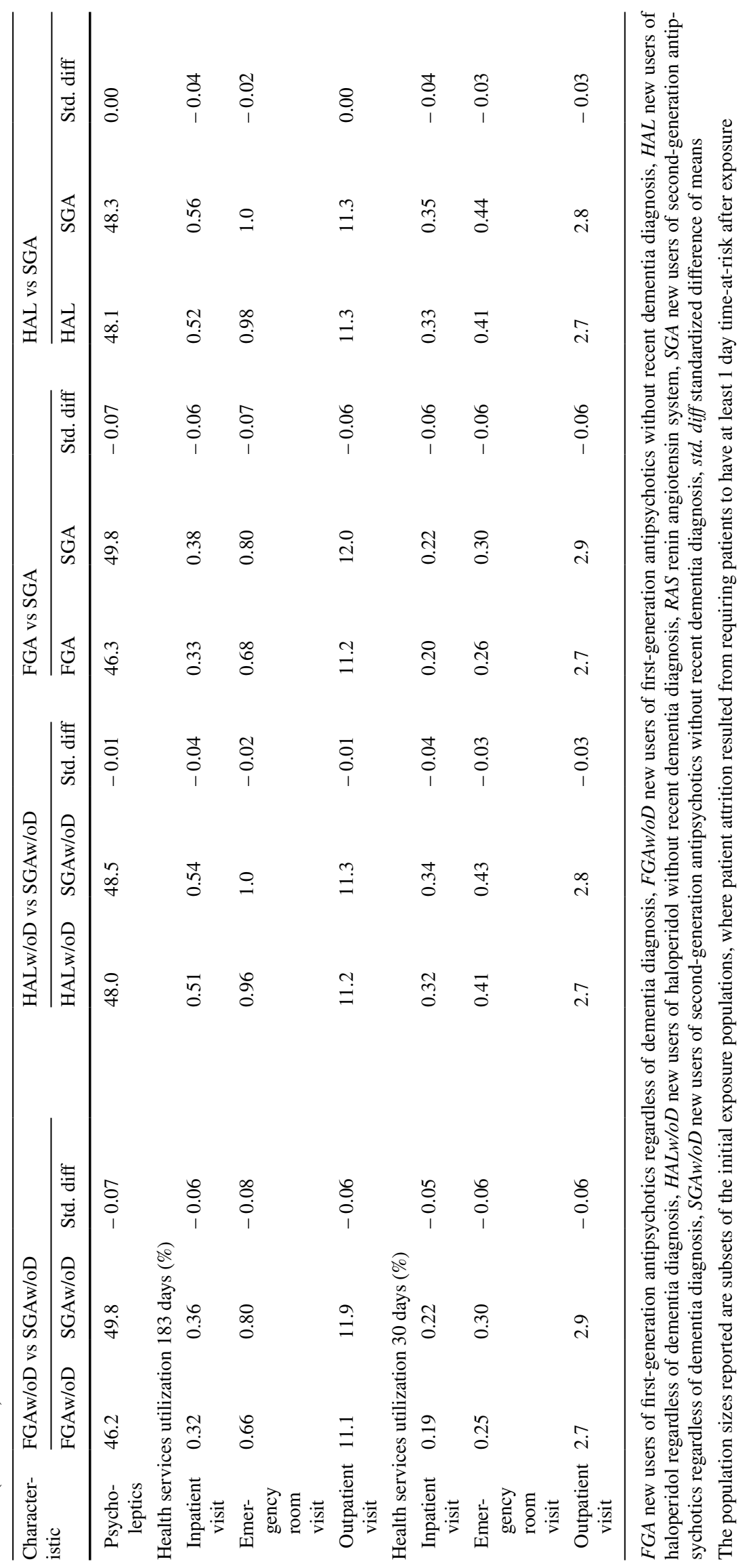



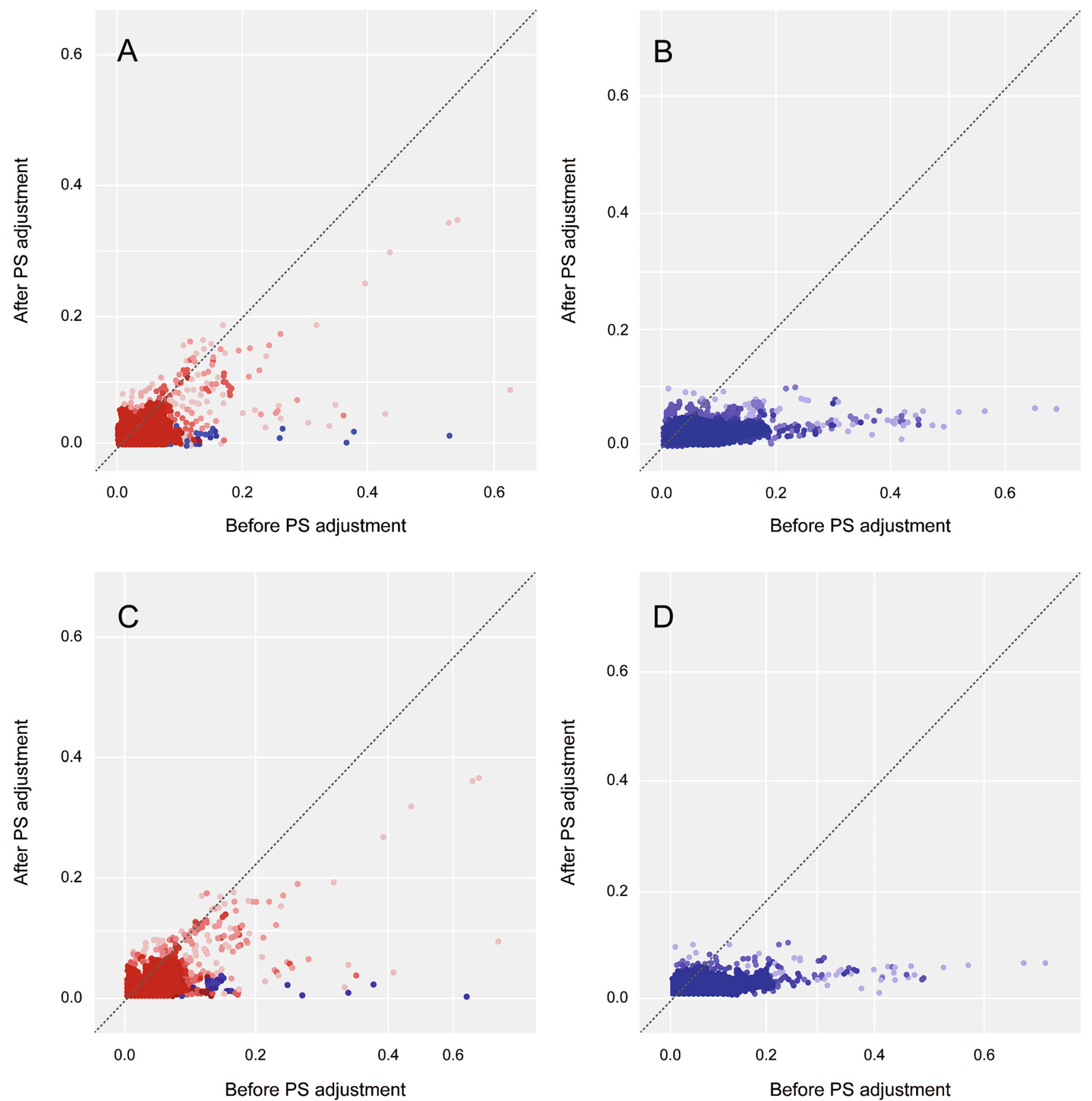

Fig. 1 Covariate balance before and after propensity score (PS) matching for new users of first-generation antipsychotics without recent dementia diagnosis $v s$ new users of second-generation antipsychotics without recent dementia diagnosis cohorts. A 1:1 PS matching of selected covariates (pre-planned analyses); B 1:10 PS matching of full covariates (pre-planned analyses); C 1:1 PS matching of selected covariates (post-hoc analyses); D 1:10 PS matching of full covariates (post-hoc analyses). Each dot represents the standardized

analyses. The corresponding estimates from the Sentinel study were $1.75(1.17-2.63)$ and $1.80(0.93-3.48)$, respectively [12]. The PS-matched HR estimates for stroke with

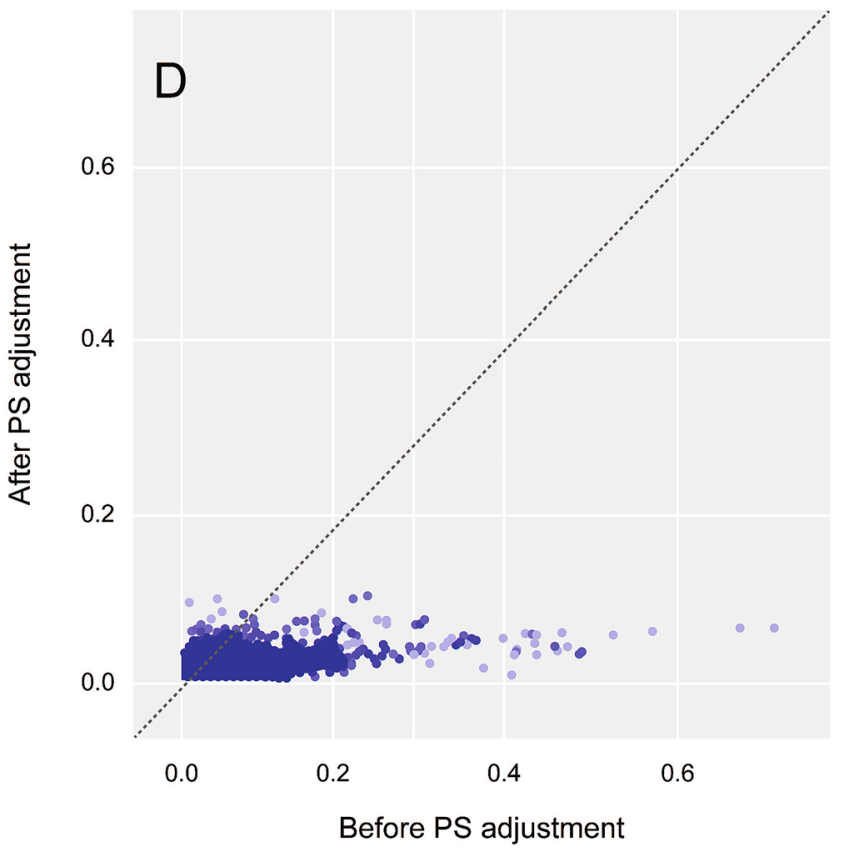

difference of means for a single covariate before and after PS adjustment. In panels $(\mathbf{A}, \mathbf{C})$, the blue data points represent the before and after matching standardized difference in means for variables that were included in the Sentinel PS model and the red data points represent covariates unadjusted for. In panels $(\mathbf{B}, \mathbf{D})$, the blue dots represent the before and after matching standardized difference in means for all observed covariates

first-generation antipsychotics and with haloperidol relative to second-generation antipsychotics in the Sentinel study were $0.87(0.54-1.41)$ and $1.31(0.54-3.21)$, respectively 

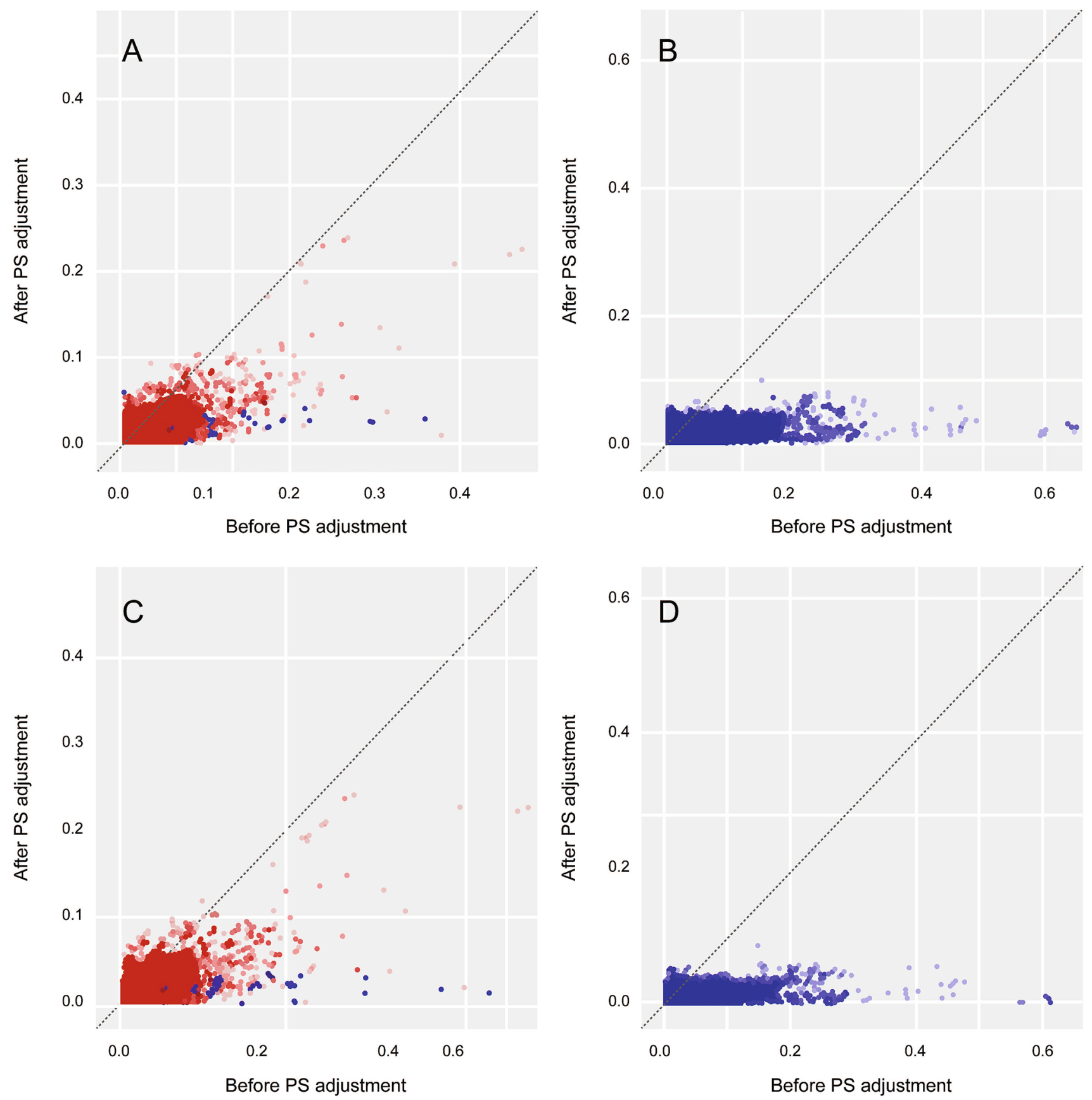

Fig. 2 Covariate balance before and after propensity score (PS) matching for new users of haloperidol without recent dementia diagnosis $v s$ new users of second-generation antipsychotics without recent dementia diagnosis cohorts. A 1:1 PS matching of selected covariates (pre-planned analyses); B 1:10 PS matching of full covariates (preplanned analyses); C 1:1 PS matching of selected covariates (posthoc analyses); D 1:10 PS matching of full covariates (post-hoc analy-

[12]. For first-generation antipsychotics vs second-generation antipsychotics, the HR in the present study (matched on the PS that mimicked the PS used in the Sentinel study) overlapped the upper end of in the CI from the Sentinel

ses). Each dot represents the standardized difference of means for a single covariate before and after PS adjustment. In panels $(\mathbf{A}, \mathbf{C})$, the blue data points represent the before and after matching standardized difference in means for variables that were included in the Sentinel PS model and the red data points represent covariates unadjusted for. In panels (B, D), the blue dots represent the before and after matching standardized difference in means for all observed covariates

study in the pre-planned analysis but not in the post-hoc analysis. For haloperidol vs second-generation antipsychotics, the lower ends of the CIs for the HR (matched on the PS that mimicked the PS used in the Sentinel study) overlapped 

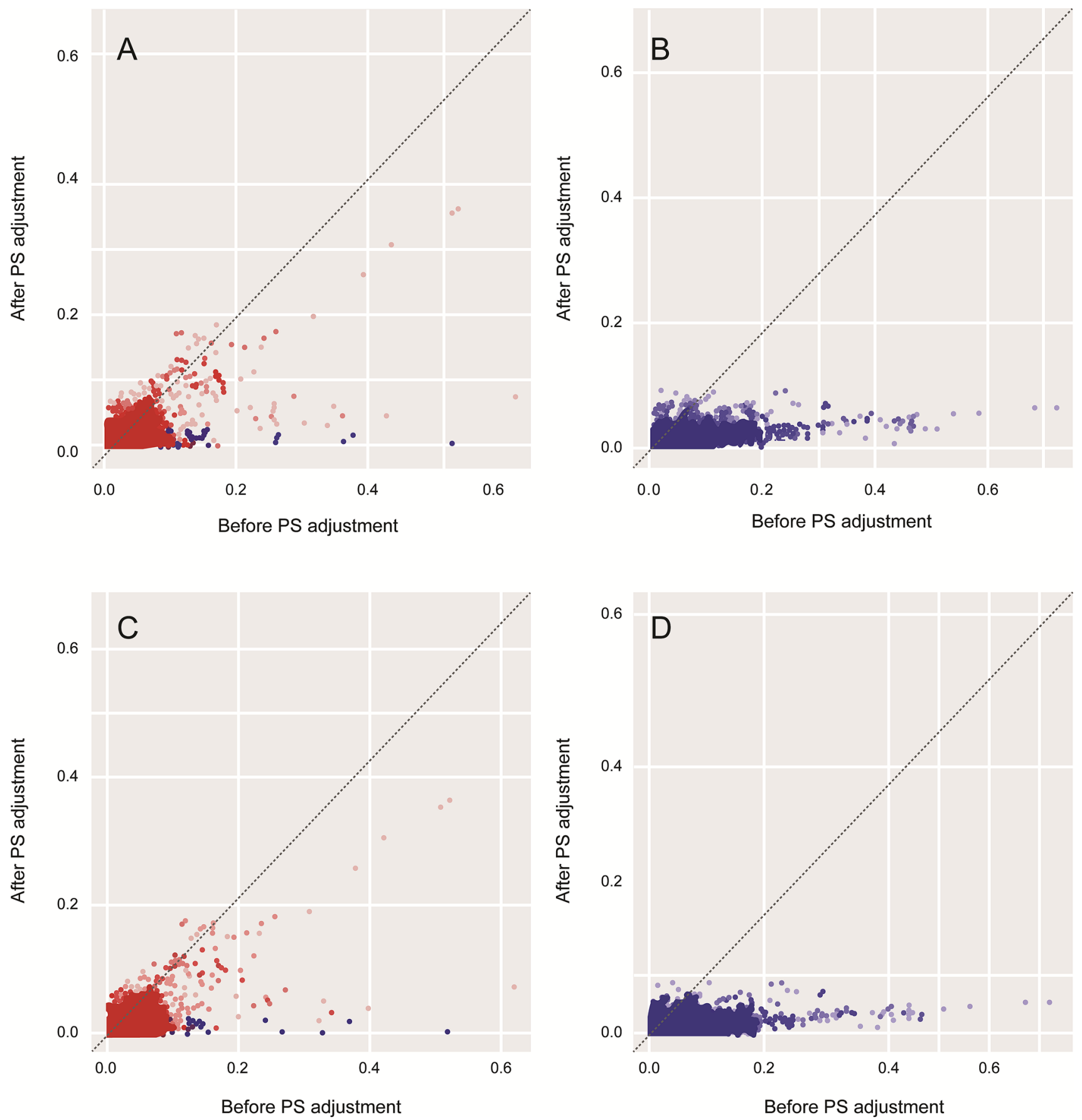

Fig. 3 Covariate balance before and after propensity score (PS) matching for new users of first-generation antipsychotics regardless of dementia diagnosis $v s$ new users of second-generation antipsychotics regardless of dementia diagnosis cohorts. A 1:1 PS matching of selected covariates (pre-planned analyses); B 1:10 PS matching of full covariates (pre-planned analyses); C 1:1 PS matching of selected covariates (post-hoc analyses); D 1:10 PS matching of full covariates (post-hoc analyses). Each dot represents the standardized differ-

ence of means for a single covariate before and after PS adjustment. In panels $(\mathbf{A}, \mathbf{C})$, the blue data points represent the before and after matching standardized difference in means for variables that were included in the Sentinel PS model and the red data points represent covariates unadjusted for. In panels $(\mathbf{B}, \mathbf{D})$, the blue dots represent the before and after matching standardized difference in means for all observed covariates 

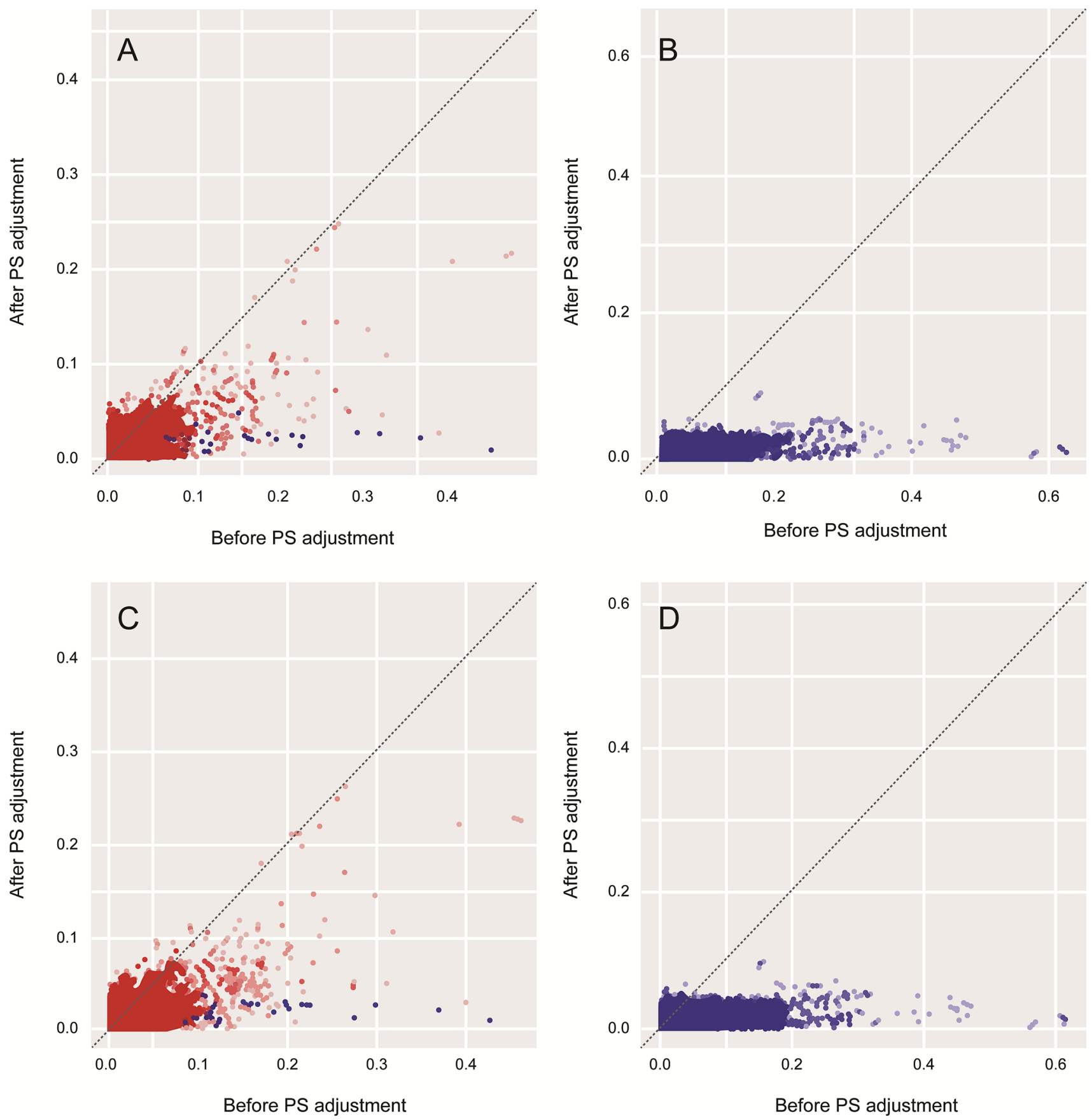

Fig. 4 Covariate balance before and after propensity score matching for new users of haloperidol regardless of dementia diagnosis $v s$ new users of second-generation antipsychotics regardless of dementia diagnosis cohorts. A 1:1 propensity score (PS) matching of selected covariates (pre-planned analyses); B 1:10 PS matching of full covariates (pre-planned analyses); C 1:1 PS matching of selected covariates (post-hoc analyses); D 1:10 PS matching of full covariates (post-hoc analyses). Each dot represents the standardized difference of means

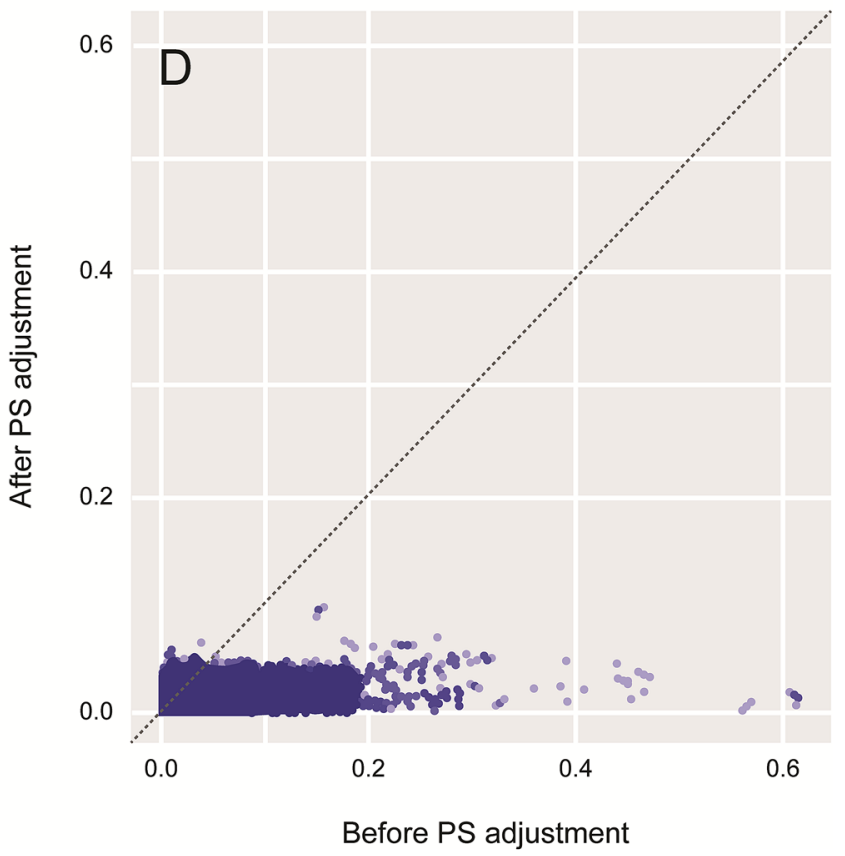

for a single covariate before and after PS adjustment. In panels (A, C), the blue data points represent the before and after matching standardized difference in means for variables that were included in the Sentinel PS model and the red data points represent covariates unadjusted for. In panels $(\mathbf{B}, \mathbf{D})$, the blue dots represent the before and after matching standardized difference in means for all observed covariates 
Table 2 Crude first post-index event IR and IP within the exposure cohorts

\begin{tabular}{|c|c|c|c|c|}
\hline Exposure cohort & Strata & Number of patients & IR/1000 PY & IP/1000 patients \\
\hline \multicolumn{5}{|c|}{ Pre-planned analyses } \\
\hline \multirow[t]{3}{*}{ FGAw/oD } & Overall & 50,819 & 3.099 & 0.846 \\
\hline & Women & 24,850 & 2.822 & 0.885 \\
\hline & Men & 25,969 & 3.455 & 0.809 \\
\hline \multirow[t]{3}{*}{ HALw/oD } & Overall & 12,460 & 5.994 & 1.605 \\
\hline & Women & 6753 & 6.169 & 1.629 \\
\hline & Men & 5707 & 5.794 & 1.577 \\
\hline \multirow[t]{3}{*}{ FGA } & Overall & 51,428 & 3.137 & 0.856 \\
\hline & Women & 25,142 & 2.793 & 0.875 \\
\hline & Men & 26,286 & 3.577 & 0.837 \\
\hline \multirow[t]{3}{*}{ HAL } & Overall & 12,886 & 6.115 & 1.63 \\
\hline & Women & 6961 & 5.988 & 1.58 \\
\hline & Men & 5925 & 6.261 & 1.688 \\
\hline \multirow[t]{3}{*}{ SGAw/oD } & Overall & 925,116 & 0.847 & 0.366 \\
\hline & Women & 561,659 & 0.849 & 0.37 \\
\hline & Men & 363,457 & 0.844 & 0.36 \\
\hline \multirow[t]{3}{*}{ SGA } & Overall & 931,800 & 0.902 & 0.391 \\
\hline & Women & 565,068 & 0.879 & 0.384 \\
\hline & Men & 366,732 & 0.937 & 0.401 \\
\hline \multicolumn{5}{|l|}{ Post-hoc analyses } \\
\hline \multirow[t]{3}{*}{ FGAw/oD } & Overall & 45,239 & 3.127 & 0.862 \\
\hline & Women & 22,548 & 2.692 & 0.843 \\
\hline & Men & 22,691 & 3.695 & 0.881 \\
\hline \multirow[t]{3}{*}{ HALw/oD } & Overall & 10,885 & 5.89 & 1.562 \\
\hline & Women & 5921 & 5.884 & 1.52 \\
\hline & Men & 4964 & 5.897 & 1.612 \\
\hline \multirow[t]{3}{*}{ FGA } & Overall & 45,789 & 3.173 & 0.874 \\
\hline & Women & 22,810 & 2.665 & 0.833 \\
\hline & Men & 22,979 & 3.833 & 0.914 \\
\hline \multirow[t]{3}{*}{ HAL } & Overall & 11,272 & 6.057 & 1.597 \\
\hline & Women & 6108 & 5.713 & 1.473 \\
\hline & Men & 5164 & 6.446 & 1.743 \\
\hline \multirow[t]{3}{*}{ SGAw/oD } & Overall & 784,719 & 0.924 & 0.389 \\
\hline & Women & 476,875 & 0.936 & 0.396 \\
\hline & Men & 307,844 & 0.906 & 0.377 \\
\hline \multirow[t]{3}{*}{ SGA } & Overall & 790,433 & 0.988 & 0.416 \\
\hline & Women & 479,805 & 0.973 & 0.413 \\
\hline & Men & 310,628 & 1.013 & 0.422 \\
\hline
\end{tabular}

$F G A$ new users of first-generation antipsychotics regardless of dementia diagnosis, $F G A w / o D$ new users of first-generation antipsychotics without recent dementia diagnosis, $H A L$ new users of haloperidol regardless of dementia diagnosis, $H A L w / o D$ new users of haloperidol without recent dementia diagnosis, $I P$ incidence proportion, $I R$ incidence rate, $P Y$ person-years, $S G A$ new users of second-generation antipsychotics regardless of dementia diagnosis, $S G A w / o D$ new users of second-generation antipsychotics without recent dementia diagnosis

The population sizes reported are subsets of the initial exposure populations, where patient attrition resulted from requiring patients to have at least 1 day of time-at-risk after exposure the upper end of the CI from the Sentinel study estimate in both the pre-planned and post-hoc analyses. Thus, the findings of the present study partially replicated the findings of the Sentinel study.
Replication of a previously conducted study provides an opportunity to increase confidence in established hypotheses, theories, or claims [36]. Replication is often subject to several challenges including, but not limited to, variation 
Table 3 PS matched risk of stroke in non-elderly patients

Pre-planned analyses

\begin{tabular}{|c|c|c|c|c|}
\hline & FGAw/oD & SGAw/oD & HR $(95 \%$ CI $)$ & Cal. HR (95\% CI) \\
\hline Unadjusted & 46,401 & 921,788 & $3.46(2.42-4.79)$ & $2.93(1.32-5.93)$ \\
\hline $1: 1$ PS match $^{\mathrm{a}}$ & 46,400 & 46,400 & $2.75(1.27-6.58)$ & $2.60(0.99-6.89)$ \\
\hline \multirow[t]{2}{*}{ 1:10 PS match ${ }^{\mathrm{b}}$} & 40,414 & 257,110 & $1.90(1.08-3.24)$ & $2.05(1.13-3.89)$ \\
\hline & HALw/oD & SGAw/oD & HR $(95 \% \mathrm{CI})$ & Cal. HR $(95 \% \mathrm{CI})$ \\
\hline Unadjusted & 10,809 & 924,041 & $6.58(3.82-10.51)$ & $8.32(2.96-19.68)$ \\
\hline $1: 1 \mathrm{PS}$ match $^{\mathrm{a}}$ & 10,809 & 10,809 & $1.80(0.62-5.86)$ & $1.92(0.53-7.82)$ \\
\hline \multirow[t]{2}{*}{ 1:10 PS match ${ }^{\mathrm{b}}$} & 10,807 & 90,637 & $2.28(1.09-4.46)$ & $2.47(1.14-5.48)$ \\
\hline & FGA & SGA & HR $(95 \%$ CI) & Cal. HR (95\% CI) \\
\hline Unadjusted & 46,934 & 928,385 & $3.28(2.31-4.53)$ & $2.77(1.24-5.66)$ \\
\hline $1: 1$ PS match $^{\mathrm{a}}$ & 46,933 & 46,933 & $2.18(1.09-4.64)$ & $2.03(0.79-5.41)$ \\
\hline \multirow[t]{2}{*}{ 1:10 PS match ${ }^{\mathrm{b}}$} & 40,983 & 262,021 & $1.52(0.90-2.50)$ & $1.64(0.94-2.97)$ \\
\hline & HAL & SGA & HR $(95 \%$ CI $)$ & Cal. HR (95\% CI) \\
\hline Unadjusted & 11,186 & 930,670 & $6.35(3.75-10.00)$ & $6.54(2.00-26.89)$ \\
\hline 1:1 PS match ${ }^{\mathrm{a}}$ & 11,186 & 11,186 & $2.20(0.80-6.98)$ & $2.10(0.67-7.90)$ \\
\hline 1:10 PS match ${ }^{\mathrm{b}}$ & 11,184 & 93,548 & $1.82(0.93-3.34)$ & $1.98(0.99-4.00)$ \\
\hline \multicolumn{5}{|c|}{ Post-hoc analyses } \\
\hline & FGAw/oD & SGAw/oD & HR $(95 \%$ CI $)$ & Cal. HR (95\% CI) \\
\hline Unadjusted & 41,427 & 781,882 & $3.11(2.13-4.38)$ & $2.50(1.14-6.85)$ \\
\hline 1:1 PS match ${ }^{\mathrm{a}}$ & 41,426 & 41,426 & $4.20(1.71-12.58)$ & $4.13(1.27-13.27)$ \\
\hline \multirow[t]{2}{*}{ 1:10 PS match ${ }^{\mathrm{b}}$} & 36,198 & 227,973 & $1.49(0.83-2.58)$ & $1.59(0.87-3.01)$ \\
\hline & HALw/oD & SGAw/oD & HR (95\% CI) & Cal. HR (95\% CI) \\
\hline Unadjusted & 9499 & 783,827 & $5.64(3.07-9.43)$ & $6.87(2.14-17.45)$ \\
\hline 1:1 PS match ${ }^{\mathrm{a}}$ & 9497 & 9497 & $4.00(1.00-26.50)$ & $4.22(0.75-28.86)$ \\
\hline \multirow[t]{2}{*}{ 1:10 PS match ${ }^{\mathrm{b}}$} & 9497 & 79,490 & $2.52(1.16-5.16)$ & $2.79(1.24-6.42)$ \\
\hline & FGA & SGA & HR (95\% CI) & Cal. HR (95\% CI) \\
\hline Unadjusted & 41,913 & 787,515 & $2.95(2.03-4.14)$ & $2.36(1.07-6.79)$ \\
\hline 1:1 PS match ${ }^{\mathrm{a}}$ & 41,912 & 41,912 & $1.27(0.65-2.53)$ & $1.11(0.44-2.85)$ \\
\hline \multirow[t]{2}{*}{ 1:10 PS match ${ }^{\mathrm{b}}$} & 36,676 & 231,410 & $1.35(0.76-2.29)$ & $1.41(0.79-2.62)$ \\
\hline & HAL & SGA & HR $(95 \%$ CI $)$ & Cal. HR (95\% CI) \\
\hline Unadjusted & 9846 & 789,488 & $5.50(3.07-9.03)$ & $5.72(1.58-23.84)$ \\
\hline 1:1 PS match ${ }^{\mathrm{a}}$ & 9845 & 9845 & $2.50(0.84-9.11)$ & $2.77(0.72-14.57)$ \\
\hline $1: 10$ PS match ${ }^{\mathrm{b}}$ & 9843 & 82,164 & $3.10(1.52-6.07)$ & $3.47(1.63-7.92)$ \\
\hline
\end{tabular}

Cal. $H R$ calibrated hazard ratio, $C I$ confidence interval, FGA new users of first-generation antipsychotics regardless of dementia diagnosis, $F G A w / o D$ new users of first-generation antipsychotics without recent dementia diagnosis, $H A L$ new users of haloperidol regardless of dementia diagnosis, $H A L w / o D$ new users of haloperidol without recent dementia diagnosis, $H R$ hazard ratio, $P S$ propensity score, $S G A$ new users of second-generation antipsychotics regardless of dementia diagnosis, $S G A w / o D$ new users of second-generation antipsychotics without recent dementia diagnosis

The population sizes reported are subsets of the initial exposure populations, where patient attrition resulted from (a) excluding patients from the second exposure cohort after switching treatment from the first, (b) requiring patients to have at least 1 day time-at-risk after exposure, and (c) matching on the PS

a 1:1 PS matching used a PS analogous to the Sentinel study PS [12]

${ }^{\mathrm{b}} 1: 10$ PS matching used the large-scale logistic regression PS

in study populations (different database, inadequate reporting of patient selection criteria, or different geographical location); presence of other confounding factors; and the difference in calendar time between the original study and the replication study can directly influence study outcomes
$[37,38]$. As noted above, the adapted PS strategy included more variables than the Sentinel PS strategy that mimicked the PS used in the Sentinel study and provided better matching on baseline covariates. Therefore, in summarizing the results of the present study, we focus on the HRs that were 
adjusted using the adapted PS strategy, and the associated calibrated CIs (cCIs).

For patients without a recent dementia diagnosis, after matching on the adapted PS strategy, the estimated HR point estimate for stroke risk among new users of first-generation antipsychotics compared to new users of second-generation antipsychotics was $>1$ and the $95 \%$ cCIs excluded 1 in the pre-planned analysis, and the point estimate was $>1$ but the cCI included 1 in the post-hoc analysis. Among new users of haloperidol vs second-generation antipsychotics, the point estimates were $>1$ and the $95 \%$ cCIs excluded 1 in both the pre-planned and post-hoc analyses. Thus, in this population, the present study found that the stroke risk associated with haloperidol exposure is higher than the stroke risk associated with second-generation antipsychotic exposure; however, the findings for first-generation vs second-generation antipsychotics are less conclusive.

Among all patients with or without a recent dementia diagnosis, after matching on the adapted PS matching strategy in both the pre-planned and post-hoc analyses, the HRs for stroke with first-generation vs second-generation antipsychotics and for haloperidol vs second-generation antipsychotics had point estimates $>1$ but all the cCIs included 1 except the cCI for haloperidol vs second-generation antipsychotics in the post-hoc analyses. Thus, in this broader population, the present study does not find an increased stroke risk for either first-generation vs second-generation antipsychotics or haloperidol vs second-generation antipsychotics.

Our study had several strengths including a large sample size, inclusion of patients from many regions of the USA, and the use of two different PS matching approaches. Among its limitations were a lack of information on socioeconomic status, health-related behaviors (including smoking and alcohol use and abuse), and body mass index. Because the database describes US residents insured through employment and their dependents, the study's finding may not generalize to other populations. In addition, because complementary drug sample usage is not captured in claims databases, for certain patients, exposure to the drug might have been classified as non-exposure or an ongoing antipsychotic medication use might have been classified as new drug use.

\section{Conclusions}

The present study found that among adults aged $\leq 64$ years, who did not have a recent diagnosis of dementia, the risk of stroke is higher among those exposed to haloperidol than those exposed to second-generation antipsychotics.

Supplementary Information The online version contains supplementary material available at https://doi.org/10.1007/s40801-021-00267-2.
Acknowledgments The authors acknowledge Gurpreet Virya and Leo J. Philip Tharappel (SIRO Clinpharm Pvt. Ltd.) for providing writing assistance and Ellen Baum, PhD (Janssen Global Services, LLC) for additional editorial support.

\section{Declarations}

Funding This study was sponsored by Janssen Research \& Development, LLC.

Conflicts of interest/Competing interests All the authors are employees of Janssen Research \& Development and own stock and stock options.

Ethics approval The use of IBM MarketScan ${ }^{\circledR}$ Commercial Database was reviewed by the New England Institutional Review Board and determined to be exempt from broad institutional review board approval, as this research project did not involve human patient research.

Consent to participate Not applicable.

Consent for publication Not applicable.

Availability of data and material The code lists used for identifying first-generation antipsychotics, haloperidol, second-generation antipsychotics, and stroke are available in the protocol, which is pre-registered at https://clinicaltrials.gov/ct2/show/NCT04002700 and is publicly available in greater detail at https://github.com/ohdsi-studies/Strok eRiskInApUsers/tree/master/documents. Results of the present study are publicly available through the interactive online tool at https://data. ohdsi.org/StrokeRiskInNonElderlyApUsers/.

Code availability The code is available at: https://github.com/ohdsistudies/StrokeRiskInApUsers.

Authors' contributions Conception and design: all authors. Collection and assembly of data: all authors but primarily CB and JW. Data analysis and interpretation: all authors but primarily CB and JW. Manuscript writing: all authors. Final approval of manuscript: all authors.

Open Access This article is licensed under a Creative Commons Attribution-NonCommercial 4.0 International License, which permits any non-commercial use, sharing, adaptation, distribution and reproduction in any medium or format, as long as you give appropriate credit to the original author(s) and the source, provide a link to the Creative Commons licence, and indicate if changes were made. The images or other third party material in this article are included in the article's Creative Commons licence, unless indicated otherwise in a credit line to the material. If material is not included in the article's Creative Commons licence and your intended use is not permitted by statutory regulation or exceeds the permitted use, you will need to obtain permission directly from the copyright holder. To view a copy of this licence, visit http://creativecommons.org/licenses/by-nc/4.0/.

\section{References}

1. Christian R, Saavedra L, Gaynes BN, et al. Appendix A, tables of FDA-approved indications for first- and second-generation antipsychotics. Future research needs for first- and second-generation 
antipsychotics for children and young adults. Rockville (MD): Agency for Healthcare Research and Quality (US); 2012: p. A1-A2.

2. Maglione M, Maher HR, Hu J, Wang Z, Shanman R, Shekelle PG, et al. Off-label use of atypical antipsychotics: an update. Comparative effectiveness review no. 43. Rockville (MD): Agency for Healthcare Research and Quality; 2011.

3. Olfson M, Blanco C, Wang S, Laje G, Correll CU. National trends in the mental health care of children, adolescents, and adults by office-based physicians. JAMA Psychiat. 2014;71:81-90. https:// doi.org/10.1001/jamapsychiatry.2013.3074.

4. Olfson M, King M, Schoenbaum M. Treatment of young people with antipsychotic medications in the United States. JAMA Psychiat. 2015;72:867-74. https://doi.org/10.1001/jamapsychiatry. 2015.0500 .

5. Driessen J, Baik SH, Zhang Y. Trends in off-label use of secondgeneration antipsychotics in the Medicare population from 2006 to 2012. Psychiatr Serv. 2016;67:898-903. https://doi.org/10.1176/ appi.ps.201500316.

6. Vijay A, Becker JE, Ross JS. Patterns and predictors of off-label prescription of psychiatric drugs. PLoS ONE. 2018;13:e0198363. https://doi.org/10.1371/journal.pone.0198363.

7. Douglas IJ, Smeeth L. Exposure to antipsychotics and risk of stroke: self controlled case series study. BMJ. 2008;337:a1227. https://doi.org/10.1136/bmj.a1227.

8. Mundet-Tuduri X, Iglesias-Rodal M, Olmos-Dominguez C, et al. Cardiovascular risk factors in chronic treatment with antipsychotic agents used in primary care. Rev Neurol. 2013;57:495-503.

9. Zivkovic S, Koh CH, Kaza N, Jackson CA. Antipsychotic drug use and risk of stroke and myocardial infarction: a systematic review and meta-analysis. BMC Psychiatry. 2019;19:189. https://doi.org/ 10.1186/s12888-019-2177-5.

10. Hsieh PH, Hsiao FY, Gau SS, Gau CS. Use of antipsychotics and risk of cerebrovascular events in schizophrenic patients: a nested case-control study. J Clin Psychopharmacol. 2013;33:299-305. https://doi.org/10.1097/JCP.0b013e3182900dfe.

11. Papola D, Ostuzzi G, Gastaldon C, et al. Antipsychotic use and risk of life-threatening medical events: umbrella review of observational studies. Acta Psychiatr Scand. 2019;140:227-43. https:// doi.org/10.1111/acps.13066.

12. Taylor LG, Panucci G, Mosholder AD, Toh S, Huang TY. Antipsychotic use and stroke: a retrospective comparative study in a non-elderly population. J Clin Psychiatry. 2019. https://doi.org/ 10.4088/JCP. $18 \mathrm{~m} 12636$.

13. Shin JY, Choi NK, Lee J, et al. A comparison of risperidone and haloperidol for the risk of ischemic stroke in the elderly: a propensity score-matched cohort analysis. J Psychopharmacol. 2015;29:903-9. https://doi.org/10.1177/0269881115578162.

14. Herrmann N, Mamdani M, Lanctot KL. Atypical antipsychotics and risk of cerebrovascular accidents. Am J Psychiatry. 2004;161:1113-5. https://doi.org/10.1176/appi.ajp.161.6.1113.

15. Laredo L, Vargas E, Blasco AJ, et al. Risk of cerebrovascular accident associated with use of antipsychotics: population-based case-control study. J Am Geriatr Soc. 2011;59:1182-7. https:// doi.org/10.1111/j.1532-5415.2011.03479.x.

16. Wang M-T, Li M-F, Chu C-L, et al. Evaluation of stroke risk associated with the use of typical or atypical antipsychotics among patients with cardiovascular diseases. J Med Sci. 2015;35:11-8. https://doi.org/10.4103/1011-4564.151284.

17. Zhang Y, Chapman AM, Plested M, Jackson D, Purroy F. The incidence, prevalence, and mortality of stroke in France, Germany, Italy, Spain, the UK, and the US: a literature review. Stroke Res Treat. 2012;2012:436125. https://doi.org/10.1155/2012/436125.

18. Cascade EF, Kalali AH, Citrome L. Antipsychotic use varies by patient age. Psychiatry (Edgmont). 2007;4:20-3.
19. Wang CC, Farley JF. Patterns and predictors of antipsychotic medication use among the U.S. population: findings from the Medical Expenditure Panel Survey. Res Social Adm Pharm. 2013;9:26375. https://doi.org/10.1016/j.sapharm.2009.07.001.

20. Shin JY, Choi NK, Lee J, et al. Risk of ischemic stroke associated with the use of antipsychotic drugs in elderly patients: a retrospective cohort study in Korea. PLoS ONE. 2015;10:e0119931. https://doi.org/10.1371/journal.pone.0119931.

21. Sultana J, Fontana A, Giorgianni F, et al. Measuring the effectiveness of safety warnings on the risk of stroke in older antipsychotic users: a nationwide cohort study in two large electronic medical records databases in the United Kingdom and Italy. Drug Saf. 2019;42:1471-85. https://doi.org/10.1007/s40264-019-00860-z.

22. Sacchetti E, Trifiro G, Caputi A, et al. Risk of stroke with typical and atypical anti-psychotics: a retrospective cohort study including unexposed subjects. J Psychopharmacol. 2008;22:3946. https://doi.org/10.1177/0269881107080792.

23. Fife D, Blacketer C, Knight RK, Weaver J. Stroke risk among elderly users of haloperidol and typical antipsychotics versus atypical antipsychotics: a real-world study from a US health insurance claims database. Am J Geriatr Psychiatry. 2020. https://doi.org/ 10.1016/j.jagp.2020.09.017.

24. US Food and Drug Administration. Haldol® brand of haloperidol injection (for immediate release). https://www.accessdata.fda. gov/drugsatfda_docs/label/2008/015923s082,018701s057lbl.pdf. Accessed 6 Apr 2021.

25. Hripcsak G, Duke JD, Shah NH, et al. Observational Health Data Sciences and Informatics (OHDSI): opportunities for observational researchers. Stud Health Technol Inf. 2015;216:574-8.

26. Voss EA, Makadia R, Matcho A, et al. Feasibility and utility of applications of the common data model to multiple, disparate observational health databases. J Am Med Inform Assoc. 2015;22:553-64. https://doi.org/10.1093/jamia/ocu023.

27. Suchard MA, Simpson SE, Zorych I, Ryan P, Madigan D. Massive parallelization of serial inference algorithms for a complex generalized linear model. ACM Trans Model Comput Simul. 2013. https://doi.org/10.1145/2414416.2414791.

28. Schuemie M, Suchard M, Ryan P. CohortMethod. https://ohdsi. github.io/CohortMethod/. Accessed 4 Apr 2021.

29. Suchard MA, Schuemie MJ, Shaddox TR, et al. Cyclops. https:// ohdsi.github.io/Cyclops/. Accessed 4 Apr 2021.

30. Bodenreider O. The Unified Medical Language System (UMLS): integrating biomedical terminology. Nucleic Acids Res. 2004;32:D267-70. https://doi.org/10.1093/nar/gkh061.

31. Tian Y, Schuemie MJ, Suchard MA. Evaluating large-scale propensity score performance through real-world and synthetic data experiments. Int J Epidemiol. 2018;47:2005-14. https://doi.org/ 10.1093/ije/dyy 120.

32. Voss EA, Boyce RD, Ryan PB, et al. Accuracy of an automated knowledge base for identifying drug adverse reactions. J Biomed Inf. 2017;66:72-81. https://doi.org/10.1016/j.jbi.2016.12.005.

33. Schuemie MJ, Ryan PB, DuMouchel W, Suchard MA, Madigan D. Interpreting observational studies: why empirical calibration is needed to correct p-values. Stat Med. 2014;33:209-18. https:// doi.org/10.1002/sim.5925.

34. Schuemie MJ, Hripcsak G, Ryan PB, Madigan D, Suchard MA. Empirical confidence interval calibration for population-level effect estimation studies in observational healthcare data. Proc Natl Acad Sci U S A. 2018;115:2571-7. https://doi.org/10.1073/ pnas. 1708282114.

35. Rothman KJ. No adjustments are needed for multiple comparisons. Epidemiology. 1990;1:43-6.

36. Nosek BA, Errington TM. What is replication? PLoS Biol. 2020;18:e3000691. https://doi.org/10.1371/journal.pbio.3000691. 
37. Madigan D, Ryan PB, Schuemie M, et al. Evaluating the impact of database heterogeneity on observational study results. Am J Epidemiol. 2013;178:645-51. https://doi.org/10.1093/aje/kwt010.

38. Amrhein V, Trafimow D, Greenland S. Inferential statistics as descriptive statistics: there is no replication crisis if we don't expect replication. Am Stat. 2019;73:262-70. https://doi.org/10. 1080/00031305.2018.1543137. 